\title{
GENERAL DISCUSSION AND SHORT CONTRIBUTIONS
}

\section{Non-Hubble Redshift in Stephan's Quintet}

C. Balkowsky, L. Bottinelli, P. Chamaraux, L. Gouguenheim, and J. Heidmann : We think it worthwhile presenting evidence at the $98.7 \%$ confidence level for the existence of a non-Hubble redshift for the galaxy NGC 7319 in Stephan's quintet.

As seen in Arp's previous paper, Stephan's quintet is a tight group of five galaxies. One of them, NGC 7320, has a redshift $c z \simeq 800 \mathrm{~km} \mathrm{~s}^{-1}$ while the four others, among them NGC 7319, have much higher redshifts $c z \simeq 6000 \mathrm{~km} \mathrm{~s}^{-1}$.

We observed NGC 7319 and 7320 in the $21-\mathrm{cm}$ line of neutral hydrogen with the Nancay radio telescope and from these observations we obtained distance estimates, without using redshifts, from distance criteria entirely independent of redshifts worked out in previous studies of 150 galaxies (Balkowsky et al.: 1973, Astron. Astrophys. 25, 319; Balkowsky: 1973, Astron. Astrophys., in press).

From the line profiles we obtain the $21-\mathrm{cm}$ line fluxes $F_{\mathbf{H}}$ and the line width $W$. Combining them with optical data on the apparent magnitudes, the apparent photometric diameters $a$ and the morphological types $T$ we can calculate integral properties such as:

- the neutral hydrogen masses $M_{\mathrm{H}} \propto F_{\mathrm{H}} D^{2}$ where $D$ is the (unknown) distance,

- the indicative total masses $M_{i} \propto a D W^{2}$.

Then, for instance, the ratios of neutral hydrogen mass to total mass are:

$$
M_{\mathrm{H}} / M_{i}=\text { (some numerical factor) } \times F_{\mathrm{H}} a^{-1} W^{-2} D .
$$

From our statistical studies we know that this ratio should have, for a given value $T$, a given value $h(T)$. Equating (1) to $h$ we obtain $D$.

We have five distance criteria of this type and the $D$ values thus obtained are shown in Figure 1 for NGC 7319, 7320, and also for the nearby large spiral NGC 7331. From the same statistical studies we know that only three of these distance criteria are independent and $\bar{D}$ is the logarithmic mean value for the first three criteria.

For NGC 7319 we get $\bar{D}=\left(22_{-9}^{+15}\right.$ m.e. $)$ Mpc. From its redshift its distance should be $D_{\text {cosmo }}=120 \mathrm{Mpc}$ for a Hubble constant $H=50 \mathrm{~km} \mathrm{~s}^{-1} \mathrm{Mpc}^{-1}$. For $H=(80 \pm 20)$ $\mathrm{km} \mathrm{s}^{-1} \mathrm{Mpc}^{-1}$ and taking into account the error on $H$ and our error on $\bar{D}$ we find that $D_{\text {cosmo }}$ is $2.2 \mathrm{rms}$ deviations different from $\bar{D}$, i.e. there is only a $1.3 \%$ chance that NGC 7319 is at $D_{\text {cosmo }}$. It therefore has an anomalous redshift at a confidence level of $98.7 \%$.

Longair: Dr Zel'dovich has asked me to summarise some of the points discussed by Dr Sargent in his review of the evidence for and against the cosmological interpretation of the redshift (see the article by Sargent in the Proceedings of IAU Symposium No. 58, Canberra).

Sargent first described the principal evidence in favour of a genuine redshiftdistance relation for galaxies. The methods involve the selection of standard candles 


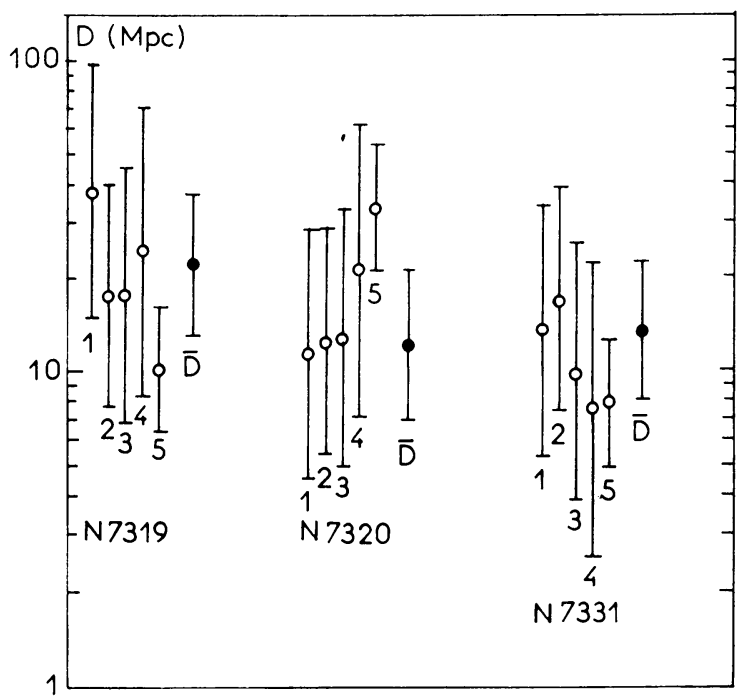

Fig. 1. Distance determinations in $\mathrm{Mpc}$ with their mean errors from five distance criteria $(1,2, \ldots, 5)$ independent of redshifts for NGC 7319, 7320 and 7331. $\bar{D}$ is the logarithmic mean of the first three criteria.

and Sargent cited (i) the redshift-magnitude relation for galaxies which are brightest cluster members (ii) the same relation for strong radio galaxies and (iii) supergiant Sc galaxies and (iv) the use of the maximum brightness of type I supernovae. A redshift-angular size correlation exists for the diameters of the brightest cluster galaxies (the work of Sandage) and for extended radio galaxies there is a redshiftangular diameter correlation in the sense that the relation has a well-defined upper bound suggesting a maximum physical extent for double radio sources. These data are strong evidence in favour of the cosmological hypothesis for the origin of the redshifts of galaxies.

Sargent discussed further data relevant to the question of the origin of redshifts of systems where non-cosmological components to their redshifts have been claimed. In the field of Stephan's quintet, there are many more galaxies with the same redshift as the four members with the larger redshift than would be expected by chance. Sargent interprets the fifth member of the quintet which has a much smaller redshift than the other four as a chance superposition of a foreground system on a background system. Further, the statistical arguments which assigned a low probability to the system being a chance superposition are weakened.

Concerning those cases where one finds bridges joining systems with grossly discrepant redshifts, Sargent suggested that the only way of demonstrating that these were not chance events was to find large numbers of these systems.

For $\mathrm{N}$ galaxies, it seems highly probable that their redshifts are wholly cosmological since Sandage has shown that there exists the same redshift-magnitude relation as for radio galaxies if one considers only the underlying galaxy and not the anomalously bright component of the emission. Further, the companion of the N-galaxy $3 \mathrm{C} 371$ and 3C 371 itself have the same redshift. 
For quasars, the cosmological interpretation can account naturally for (i) the upper envelope of the redshift-magnitude relation, (ii) the existence of a redshift-magnitude relation for various sub-sets of the quasar population (the work of Setti and Woltjer), (iii) Kristian's discovery of galaxy-like images around those quasars where it would be possible to observe them and (iv) of 7 candidates in which quasars lie in the field of a cluster of galaxies, in 5 cases the quasar and cluster have the same redshift and the two remaining cases have given no result (the work of Gunn and his colleagues).

Burbidge now believes that the peaks in the redshift distribution for quasars at 1.95 and for emission line objects at 0.061 are not statistically significant. Of the reported periodicities in the redshift distribution of emission line objects, only a periodicity of 0.031 in redshift survives and it is associated with non-quasar objects. For the latter objects, the independent evidence favours the cosmological interpretation of their redshifts.

\section{The Deceleration Parameter Based on the Redshift-Diameter Relation}

W. Baum: For determining the deceleration parameter $q$, there is an important observable relation that has not yet been mentioned here. This is the redshift-diameter relation for galaxies belonging to clusters. It provides an independent determination of $q$ that seems likely to be less vulnerable than the redshift-magnitude relation to evolutionary changes or to any imperfect transparency of space. In the next ten years, the redshift-diameter relation could well become one of our most powerful cosmological tests with the aid of expected advances in image deconvolution, image digitization, and space telescope performance.

It would not be possible to compare the metric diameter of a galaxy in one cluster with that of a galaxy of similar rank in another, if their luminosity profiles (surface brightness as a function of radial distance from the nucleus) followed a simple power law, such as inverse square or inverse cube. A ratio of diameters would then be inseparable from a ratio of surface brightness. Fortunately, the luminosity profiles of galaxies have an actual shape like that shown schematically in Figure 2, in which a horizontal displacement $A B$ of the profiles can be distinguished from a vertical one $B C$. The displacement $A B$ represents logarithmically the ratio of metric diameters, while $A D$ represents the ratio of isophotal diameters.

Observationally, the trick is to measure $A B$ by a method that permits precise removal of the instrumental profile (including 'seeing') and that is insensitive to the relative amounts of sky background radiation on which the galaxies are superimposed. In principle, these conditions were fulfilled by a simple experiment I made a few years ago at Palomar(W. A. Baum: 1972, in D. S. Evans (ed.), 'External Galaxies and Quasi Stellar Objects', IAU Symp. 44, 393-396). My measurements of $A B$ favor a $q$ less than 0.5 , but more clusters need to be measured and other techniques also ought to be explored.

Oke: I want to describe briefly a program which Dr J. E. Gunn and I have been pursuing for more than a year to extend the Hubble relation beyond $z=0.20$. The 


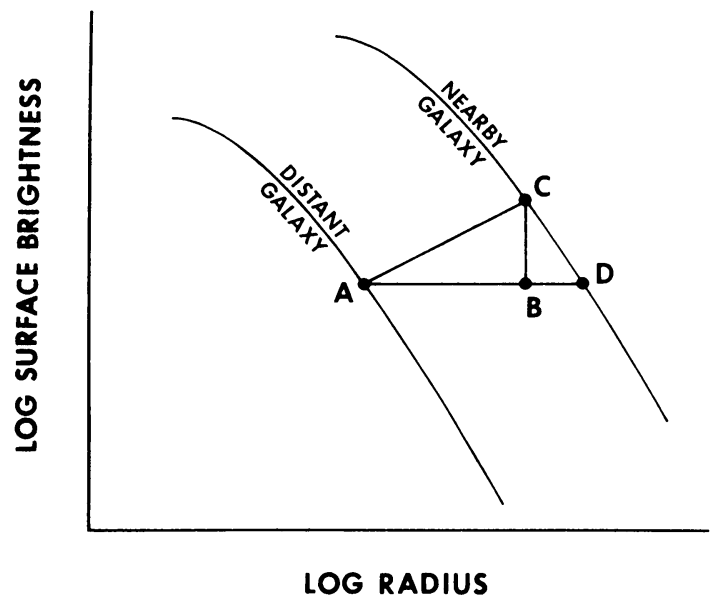

Fig. 2.

first part of the program is to discover in a statistically valid way new distant clusters of galaxies. For this purpose several 48 in. Schmidt fields $\left(6^{\circ} \times 6^{\circ}\right)$ have been photographed with IIIa-J plates. Each field contains up to 50 candidate clusters. Redshifts of galaxies in some of these clusters indicate that clusters found in this way always have $z<0.30$. To go to fainter limiting magnitudes, a survey of parts of the $6^{\circ} \times 6^{\circ}$ fields is being made with a large image tube at the prime focus of the 200 -in. telescope. With IIIa-J plates limiting exposures are obtained in $5 \mathrm{~min}$. Several very distant clusters have already been discovered but not yet studied.

The second part of the program is to use the multichannel spectrometer and new spectrometer devices to obtain redshifts and energy distributions of the brightest members of all clusters discovered in the program outlined above. Particular care will be taken to ensure that the sample is a proper one.

At the present time redshifts and energy distributions have been obtained for eighteen cluster galaxies in the redshift range $0.20<z \leqslant 0.46$. Some of the clusters are from the above survey; others are clusters near quasars. Three of the clusters have been known for many years.

Abell: Are the 18 different galaxies you have redshifts for in 18 different clusters?

Oke: There are redshifts for 18 different galaxies in 16 clusters.

Abell: The apparent connections between galaxies described by Arp may not necessarily be regarded as caused by tidal interactions. A tidal force is a differential force across the perturbed body, and in the first order the tidal distortion is symmetrical, producing effects on the perturbed body both on the side of the perturbing body and on the opposite side. Toomre discussed this point very well in Sydney (see Toomre: 1974, in J. R. Shakeshaft (ed.), 'The Formation and Dynamics of Galaxies', IAU Symp. 58, in press).

Arp: Toomre actually commented that if the companion was not responsible for the distortion and tail in NGC 7603 he did not know what was! Actually counter- 
tides depend on the kind of encounter and time scale. But most of all you are forgetting that I conclude that these companions are ejected from the center of the larger galaxy. In that case you would expect single bridges and jets to point to the companions.

Ozernoy: If the relation between the angular distance, $\theta$, of a compact object from a galaxy and the Galaxy redshift, $z_{\mathrm{G}}$, suggested by Burbidge, O'Dell and Strittmatter for the small sample of objects is real, it is reasonable to assume that it will retain its validity for a more complete sample of objects. However, as can be seen from the figure, it does not. The objects presented in Figure 3 are listed in my paper published in Astron. Zh.: 1972, 49, 1178 and include, in addition to the objects of Burbidge et al. (Nos. 1-5), both the wide pairs (Nos. 21-25), which Rowan-Robinson and Arp assume to be physically connected (although in that case the distance between the components turns out to be greater than 1-10 Mpc), and 'close' pairs (Nos. 6-20)

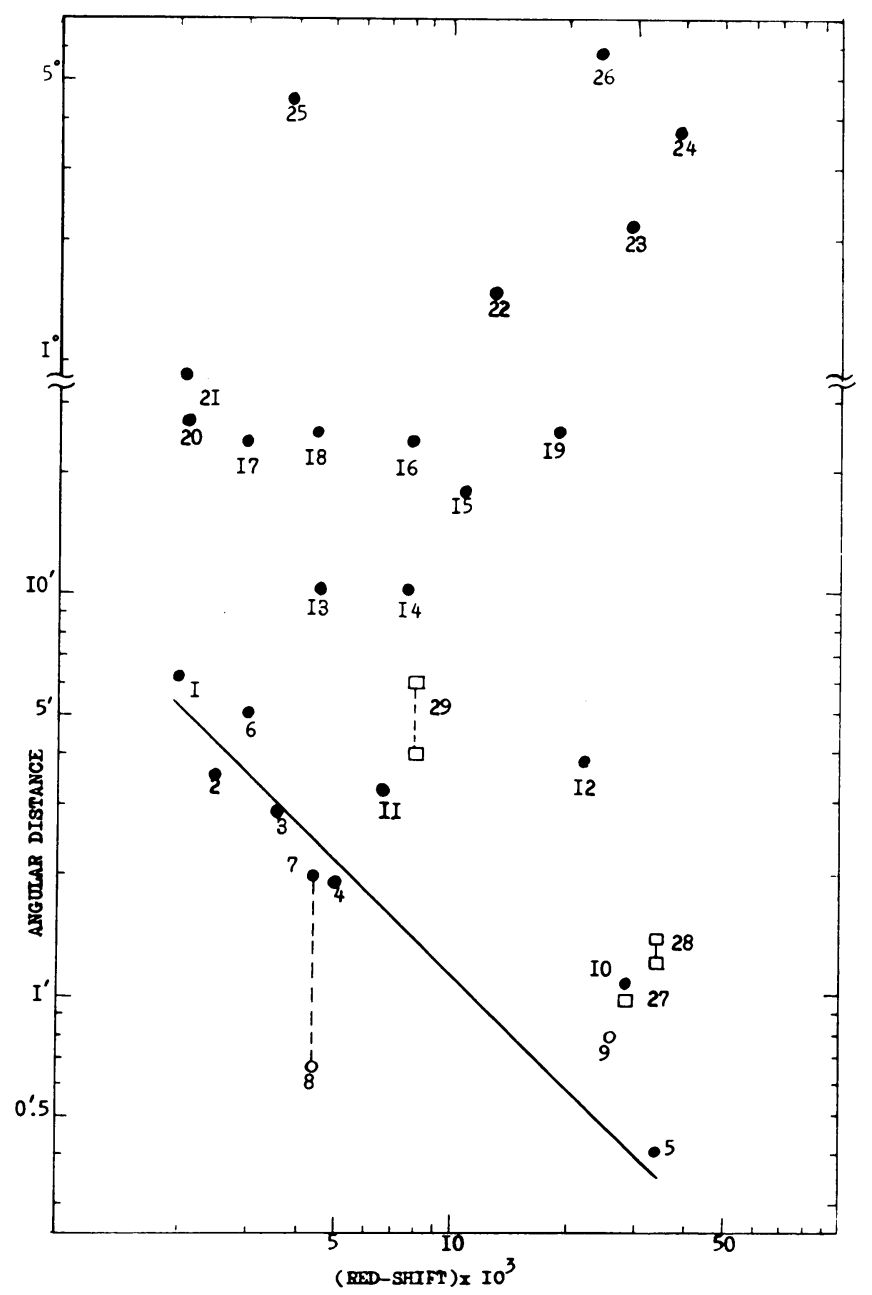

Fig. 3. 
for which $\theta<25^{\prime}$. As can be seen, even in the sample of only close pairs there is no definite relation between $\theta$ and $z_{\mathrm{G}}$. Therefore, the dependence shown on the figure by the heavy line is produced by a game of chance in an incomplete sample.

Arp: If you go further away from bright galaxies you will find an increasing percentage of non associated QSRs. This is apparently what you have done. In order to discuss the point practically you would have to produce a table of QSRs and associated galaxies, as I have done, and compute probabilities as Burbidge et al. have done.

Zel'dovich: Tammann has given the argument that $\Omega \ll 1$ as deduced from the rather exact agreement of the local form of Hubble's law in regions with rather large departures from uniformity with that derived from more distant galaxies. This argument can be put into high-brow form : in the theory of perturbations of Friedmann world models, if $\Omega=1$, both $\delta \varrho / \varrho$ and peculiar velocities $u_{\text {pec }}$ grow as powers of $t, \delta \varrho / \varrho \propto t^{2 / 3}, u_{\text {pec }} \propto t^{1 / 3}$. But if $\Omega \ll 1$ then at some redshift $(1+z) \sim 0.5 / \Omega$ the law is changed; after this moment $\delta \varrho / \varrho=$ const, the density contrast is frozen, but $u_{\text {pec }} \propto t^{-1}$ is decreasing which corresponds exactly to Tammann's conjecture.

Rees: While agreeing with Dr Zel'dovich's last remark, it may be worthwhile adding the proviso that the lack of substantial deviations from Hubble's law within the local 'supercluster' implies a low $q_{0}$ only if the 'missing mass' is presumed to be clumped to the same extent as the visible matter. One cannot use this argument to rule out a high $q_{0}$ if the missing matter is in some non-interacting relativistic form, for instance, neutrinos or gravitational waves.

Matilla: The extragalactic background brightness in the optical part of spectrum is an observational quantity of fundamental interest in several fields of cosmology. Questions involved are the decision between the various cosmological models, the existence of luminous stellar matter between the galaxies, the emission of intergalactic gas and evolutionary effects in the luminosity and number of galaxies.

The integrated light of known galaxies has been estimated using galaxy counts, and the resulting background brightness at $4000 \AA$ is close to $1.2 \times 10^{-9} \mathrm{erg} \mathrm{cm}^{-2} \mathrm{~s}^{-1}$ $\mathrm{sr}^{-1} \AA^{-1}$ for a variety of different cosmological models (Peebles and Partridge: 1967, Astrophys. J. 148, 713).

The measurement of such a small extragalactic background brightness is extremely difficult because it is swamped by other, much larger components of the night-sky brightness. In order to find the extragalactic background brightness I have carried out photoelectric observations of the night-sky brightness in the area of the dark nebula L134, which is situated at a high galactic latitude $\left(b=36^{\circ}\right)$. The method is based on the argument that the extragalactic light is strongly attenuated in the direction of an opaque dark nebula. When the dark nebula is at a sufficiently high galactic latitude the extragalactic light is only weakly attenuated in the vicinity of the dark nebula. The difference in surface brightness between the dark nebula and its surroundings is due to two components only: (1) extragalactic light, and (2) diffuse scattered starlight from interstellar dust. By making observations in two pass bands, at $3800 \AA$ and $4200 \AA$, the diffuse scattered light can be separated, and the extragalactic component is ob- 
tained. Its value in the vicinity of $\mathrm{L} 134$ is found to be $(+14.5 \pm 6) \times 10^{-9} \mathrm{erg} \mathrm{cm}^{-2} \mathrm{~s}^{-1}$ $\mathrm{sr}^{-1} \AA^{-1}$, which is more than ten times larger than the theoretically predicated value for the integrated light of known galaxies.

If the large value of the extragalactic background brightness suggested by these observations can be confirmed by further work the cosmological consequences will be far-reaching. Either a much higher value for the local luminosity density must be accepted, or the 'standard' cosmological models, used in the calculation of the integrated light of known galaxies, must be revised. If the above result of my observations is interpreted in terms of a higher local luminosity density, then also the mass density of the Universe should be substantially increased. 